\title{
Reliability Evaluation of the Design Space of the Granulation Process of Mefenamic Acid Tablets Using a Bootstrap Resampling Technique
}

\author{
Hiroaki Arai, ${ }^{* a}$ Tatsuya Suzuki, ${ }^{a}$ Shuichi Yada, ${ }^{a}$ Chosei Kaseda, ${ }^{b}$ Yoshinori OnUKi, ${ }^{c}$ and \\ Kozo TAKAYAMA ${ }^{c}$ \\ ${ }^{a}$ Formulation Technology Research Laboratories, Daiichi Sankyo Co., Ltd.; 1-12-1 Shinomiya, Hiratsuka, Kanagawa \\ 254-0014, Japan: ${ }^{b}$ Research and Development Headquarters, Yamatake Corporation; 1-12-2 Kawana, Fujisawa, \\ Kanagawa 251-8522, Japan: and ${ }^{c}$ Department of Pharmaceutics, Hoshi University; 2-4-41 Ebara, Shinagawa-ku, Tokyo \\ 142-8501, Japan. $\quad$ Received December 14, 2010; accepted February 19, 2011; published online February 22, 2011
}

The design space of the granulation process of mefenamic acid tablets, based on Box and Behnken design datasets, was described by a response surface method incorporating multivariate spline interpolation. The reliability of the optimal solutions and the acceptance ranges were evaluated by a bootstrap (BS) resampling technique. The distribution of the BS optimal solutions was almost symmetrical; however, several solutions, which were quite different from the original solution, were mixed. The reason for this problem was considered to be the mixing of the global and the local optima. Therefore, we applied self-organizing map (SOM) clustering for dividing data into several clusters and identified the cluster containing the global optima. The accuracy and reproducibility of the optimal solution in the cluster containing the optimal solution were quantitatively evaluated. In addition, the response surfaces modeled from all the BS datasets contained in the cluster were plotted into the same coordinates with the original response surface. The plots of BS optimal solutions were distributed around the original solution. Moreover, the average of all the BS response surfaces sufficiently corresponded with the original response surface. The conservative limits of the $95 \%$ confidence intervals of the acceptance ranges in three response variables could be calculated using the standard deviations of the BS response surfaces. Consequently, it was considered that a novel evaluation method based on BS resampling and SOM could be used for quantitatively evaluating the precision of the nonlinear response surface model.

Key words response surface method; quality-by-design; optimization; self-organizing map; formulation; high-shear granulation

In recent years, the "quality by design $(\mathrm{QbD})$ " concept has been introduced in the Q8 guideline of the International Conference on Harmonisation (ICH). ${ }^{1)}$ This guideline has necessitated the establishment of a science-based rationale in pharmaceutical formulation development. In particular, this guideline has recommended to establish a design space, multi-dimensional combination of input variables and process parameters that have been demonstrated to provide assurance of quality, in formulation optimization and manufacturing process estimation. The design space describes the relationship between several process inputs (material attributes and process parameters) and critical quality attributes (CQAs). Therefore, it is necessary to establish a design space to identify multidimensional combinations and interactions of the many causal factors that determine the quality of the target. ${ }^{2)}$ When describing a design space, a design of experiments (DOE) is effectively used for determining the relationship between factors affecting a process and the output of the process. ${ }^{3,4)}$ Moreover, a response surface method (RSM) is useful for the visual understanding of factors and the facilitation of the clarification of problems to be solved for optimization in a pharmaceutical development study. ${ }^{5-11)}$ The design space is represented by the response surface model resolved at the limit of a satisfactory response, and it is determined from the region of successful operating ranges for multiple critical quality attributes. Furthermore, the overlaps of some response surface models for multiple quality attributes enable the generation of a common design space with successful operating ranges. ${ }^{1)}$ Thus, working within the design space is not considered as a post approval change.

Therefore, it is important to evaluate the reliability of the design space, especially the acceptable range of input param- eters. The reliability of the classical response surface model using quadratic polynomial equations can be evaluated by a statistical analysis. However, the predictions based on the quadratic polynomial model often exhibit poor estimation in nonlinear complex problem. To resolve the nonlinear problem, computer-based artificial intelligence techniques have been used for RSM modeling in recent times. ${ }^{12-14)}$ On the other hand, the reliability of the nonlinear response surface estimated by a computer-based method cannot be directly evaluated using a conventional mathematical method. Thus, we applied a bootstrap (BS) resampling technique ${ }^{15)}$ to evaluate the reliability of the optimal solutions predicted by RSM incorporating multivariate spline interpolation $\left(\mathrm{RSM}^{\mathrm{S}}\right){ }^{14)} \mathrm{We}$ previously reported that the novel evaluation method based on a BS technique was suitable for evaluating the accuracy and precision of the optimal solution. ${ }^{16)}$ Moreover, we confirmed that self-organizing map (SOM) clustering ${ }^{17)}$ reinforced the applicability of the novel evaluation method. ${ }^{18)}$ Therefore, we quantitatively evaluated the reliability of the response surface and the design space predicted by $\mathrm{RSM}^{\mathrm{S}}$ using the BS and SOM techniques. In this study, we used an experimental dataset of mefenamic acid tablets prepared by a high-shear granulation method.

\section{Theoretical}

BS Resampling The BS resampling technique is a computer-based technique used for estimating the standard error of an empirical distribution of an observed sample. ${ }^{15,19-22)}$ Let $x=\left(x_{1}, \cdots, x_{n}\right)$ be an $n$ sample with an unknown distribution function $F$ depending on an unknown real parameter $\theta$. The problem is to evaluate parameter $\theta$ by a statistic $\hat{\theta}=s(x)$ from sample $x$ and evaluate the estimation accuracy when 
distribution $F$ is unknown. In order to evaluate the estimation accuracy, $B$ samples were generated from the initial sample $x$ by resampling. These samples were called BS samples and denoted by $x^{* b}$.

A BS sample $x^{* b}=\left(x_{1}^{* b}, \cdots, x_{n}^{* b}\right)$ was generated by random resampling by replacing the initial sample $x$. The distribution function of a BS sample $x^{* b}$ is $\hat{F}$, which is the same as the empirical distribution of $x$. A BS replicate of estimator $\widehat{\theta}=s(x)$ is $\hat{\theta}^{* b}=s\left(x^{* b}\right)$. Therefore, for the mean of sample $x$, the estimator is $s(x)=(1 / n) \sum_{i=1}^{n} x_{i}$, and a BS replicate is $s\left(x^{* b}\right)=(1 / n) \sum_{i=1}^{n} x_{i}^{* b}$.

Then, the BS estimate of the standard deviation of $\hat{\theta}$ denoted by $\sigma_{\text {boot }}(\bar{\theta})$ is given by Eqs. 1 and 2 as follows:

$$
\begin{aligned}
& \hat{\sigma}_{\text {boot }}\left(\hat{\theta}^{*}\right)=\left[\frac{1}{B-1} \sum_{b=1}^{B}\left(\hat{\theta}^{* b}-\hat{\theta}^{*}(\cdot)\right)^{2}\right]^{1 / 2} \\
& \hat{\theta}^{*}(\cdot)=\frac{1}{B} \sum_{b=1}^{B} \hat{\theta}^{* b}
\end{aligned}
$$

Novel reliability evaluation procedures for a response surface and an optimal solution have been applied in this technique.

Self-Organizing Map A self-organizing map (SOM) is a feedforward neural network that implements a nonlinear projection from high-dimensional input vectors onto a lowdimensional (typically two-dimensional) array of nodes, called a map. ${ }^{17,23)}$ At time $t$, each neuron $k$ is characterized by the reference vector $m_{k}(t)=\left[m_{i 1}(t), m_{i 2}(t), \cdots, m_{i n}(t)\right]$ and a position in low-dimensional (typically two-dimensional) nodes represented by the vector $w_{i}(t)=\left[w_{i 1}(t), w_{i 2}(t), \cdots, w_{i n}(t)\right]$. Each input vector $x(t)=\left[x_{1}(t), x_{2}(t), \cdots, x_{n}(t)\right]$ is compared with each reference vector, and the winner vector is considered to be that closest to the stimulus. The weights of the weight vectors in the neighborhood of the winner vector are adjusted with a strength $\gamma(v, t)$ that is proportional to their distance to the winner vector, $v$. The neighborhood function $\gamma(v, t)$ is typically Gaussian with standard deviation $\sigma$. Winner vectors are adjusted on the basis of the following equation.

$$
m_{k}(t+1)=m_{k}(t)+\alpha(t) \gamma(v, t)\left[x(t)-m_{k}(t)\right]
$$

where $\alpha(t)$ is the algorithm's learning rate. Both $\alpha(t)$ and $\sigma(t)$ typically decrease exponentially during the ordering phase and then decrease linearly in the convergence phase.

\section{Experimental}

Materials The formulation of mefenamic acid tablets is shown in Table 1. Mefenamic acid (Daiichi Sankyo Chemical Pharma Co., Japan), lactose monohydrate (200-mesh grade, DMV International, Netherlands), microcrystalline cellulose (Ceolus ${ }^{\circledR}$ PH-101, Asahi Kasei Chemicals, Japan), lowsubstituted hydroxypropylcellulose (L-HPC ${ }^{\circledR}$ (LH-21), Shin-Etsu Chamical Co., Japan), hydroxypropylcellulose (HPC-L ${ }^{\circledR}$, Nippon Soda Co., Japan), and magnesium stearate (Nitto Kasei Kogyo K.K., Japan) were all of grades conforming to the current 'Japanese Pharmacopoeia' (JP).

Preparation Method of Sample Tablets Mefenamic acid was milled using an impact mill (Fine impact mill 100 UPZ, Hosokawa Micron Corp., Japan) with an impeller speed of $10000 \mathrm{rpm}$. The milled mefenamic acid (volume mean diameter approximately $9.5 \mu \mathrm{m}$ ), lactose monohydrate, lowsubstituted hydroxypropylcellulose, microcrystalline cellulose, and hydroxypropylcellulose were blended in a high-shear mixer (NMG-1L, Nara Machinery Co., Japan) for 2 min (impeller rotation speed was set at $700 \mathrm{rpm}$ ). After blending, water was added to the mixture, and that was kneaded continuously in a high-shear mixer. The granulation conditions are listed in Table 2, and the chopper rotation speed was set at $1500 \mathrm{rpm}$. The wet mass was dried in a fluid-bed dryer (Flow Coater Mini, Freund Corp., Japan) up to $40{ }^{\circ} \mathrm{C}$ for the exhaust air temperature (inlet air temperature was set at $80^{\circ} \mathrm{C}$ ). The coarse granules were screened in a screening mill (Quadro Comill,
Table 1. Components and Compositions of Mefenamic Acid Tablets

\begin{tabular}{lc}
\hline \hline \multicolumn{1}{c}{ Components } & Quantity $(\mathrm{mg} /$ tablet $)$ \\
\hline Mefenamic acid & 25.0 \\
Lactose monohydrate & 49.5 \\
Microcrystalline cellulose & 5.0 \\
Low-substituted hydroxypropylcellulose & 15.0 \\
Hydroxypropylcellulose & 5.0 \\
Magnesium stearate & 0.5 \\
\hline Total & 100.0 \\
\hline
\end{tabular}

Table 2. Granulation Conditions of Mefenamic Acid Tablets with Box and Behnken Experimental Design

\begin{tabular}{crrrrrr}
\hline \hline \multirow{2}{*}{$\begin{array}{c}\text { Experiment } \\
\text { number }\end{array}$} & \multicolumn{2}{c}{ Factor level } & $\begin{array}{c}\text { Amount of } \\
\text { water added } \\
(\%), X_{1}\end{array}$ & $\begin{array}{c}\text { Impeller } \\
\text { rotation speed } \\
(\mathrm{rpm}), X_{2}\end{array}$ & $\begin{array}{c}\text { Kneading } \\
\text { time } \\
(\mathrm{min}), X_{3}\end{array}$ \\
\cline { 2 - 4 } 1 & $X_{1}$ & $X_{2}$ & $X_{3}$ & & & \\
2 & 0 & -1 & 1 & 40 & 700 & 10 \\
3 & 0 & 1 & 1 & 40 & 100 & 10 \\
4 & 1 & 0 & 1 & 50 & 700 & 10 \\
5 & -1 & -1 & 0 & 30 & 100 & 10 \\
6 & -1 & 1 & 0 & 30 & 1300 & 6 \\
7 & 1 & -1 & 0 & 50 & 100 & 6 \\
8 & 1 & 1 & 0 & 50 & 1300 & 6 \\
9 & -1 & 0 & -1 & 30 & 700 & 6 \\
10 & 0 & -1 & -1 & 40 & 100 & 2 \\
11 & 0 & 1 & -1 & 40 & 1300 & 2 \\
12 & 1 & 0 & -1 & 50 & 700 & 2 \\
13 & 0 & 0 & 0 & 40 & 700 & 6 \\
14 & 0 & 0 & 0 & 40 & 700 & 6 \\
\hline
\end{tabular}

Powrex Corporation, Japan) with an open mesh screen of $1.1 \mathrm{~mm}$ in diameter and an impeller speed of $2200 \mathrm{rpm}$. The screened granules were lubricated with magnesium stearate and blended in a V-shaped blender (Type S-5, Tsutsui Scientific Instruments Co., Ltd., Japan) for $10 \mathrm{~min}$ (rotation speed was set at $51 \mathrm{rpm}$ ). The final blend was compressed into tablets using a universal testing machine (Autograph AG-20kNI, Shimadzu Co., Japan) and a round-shaped punch with double radii, $6.5 \mathrm{~mm}$ in diameter. The compression force was conducted at approximately $8 \mathrm{kN}$.

In this study, the amount of water added $\left(X_{1}\right)$, impeller rotation speed $\left(X_{2}\right)$, and kneading time $\left(X_{3}\right)$ were selected as causal factors of the granulation process. These variables were assigned according to the Box and Behnken design DOE. We prepared 14 batches of mefenamic acid tablet formulations under various manufacturing conditions.

Determination of Response Variables The dissolution ratio of mefenamic acid for the first $15 \mathrm{~min}\left(Y_{1}\right)$, hardness of the core tablets $\left(Y_{2}\right)$, and particle size of the screened granules $\left(Y_{3}\right)$ were selected as the response variables that were to be evaluated for the resulting tablets and granules. The values of these variables were obtained from the mean of 3 determinations.

a) Dissolution: Dissolution testing was performed by a paddle method according to the recommendations of the current JP at $50 \mathrm{rpm}$ in $900 \mathrm{ml}$ of phosphate buffer solution ( $\mathrm{pH} 6.8$ ) containing $2 \%$ sodium lauryl sulfate at $37^{\circ} \mathrm{C}$. The dissolved mefenamic acid was assayed by an automated flowthrough UV spectrophotometric method at $285 \mathrm{~nm}$ with a $10-\mathrm{mm}$-long cell (Automated dissolution apparatus, Toyama Sangyo Co., and Shimadzu Co., Japan).

b) Hardness: The hardness of the resulting tablets was measured using a hardness tester (Tablet tester PTB 302, Pharma Test Apparatebau AG, Austria).

c) Particle Size of the Screened Granules: The particle size of the screened granules was measured using an automated sieving apparatus (Robot Sifter RPS-85P, Seishin Enterprise Co., Japan) with 20, 32, 48, 65, 100,150 , and 200 mesh sieves. The particle size distribution and the median diameter were calculated by the ratio of the residual weight of the granules on each sieve.

The experimental dataset was prepared according to the Box and Behnken design DOE, which consisted of 3 causal factors and 3 response variables with 14 batches of mefenamic acid tablets. The dataset is named Original dataset, and shown in Table 3. 


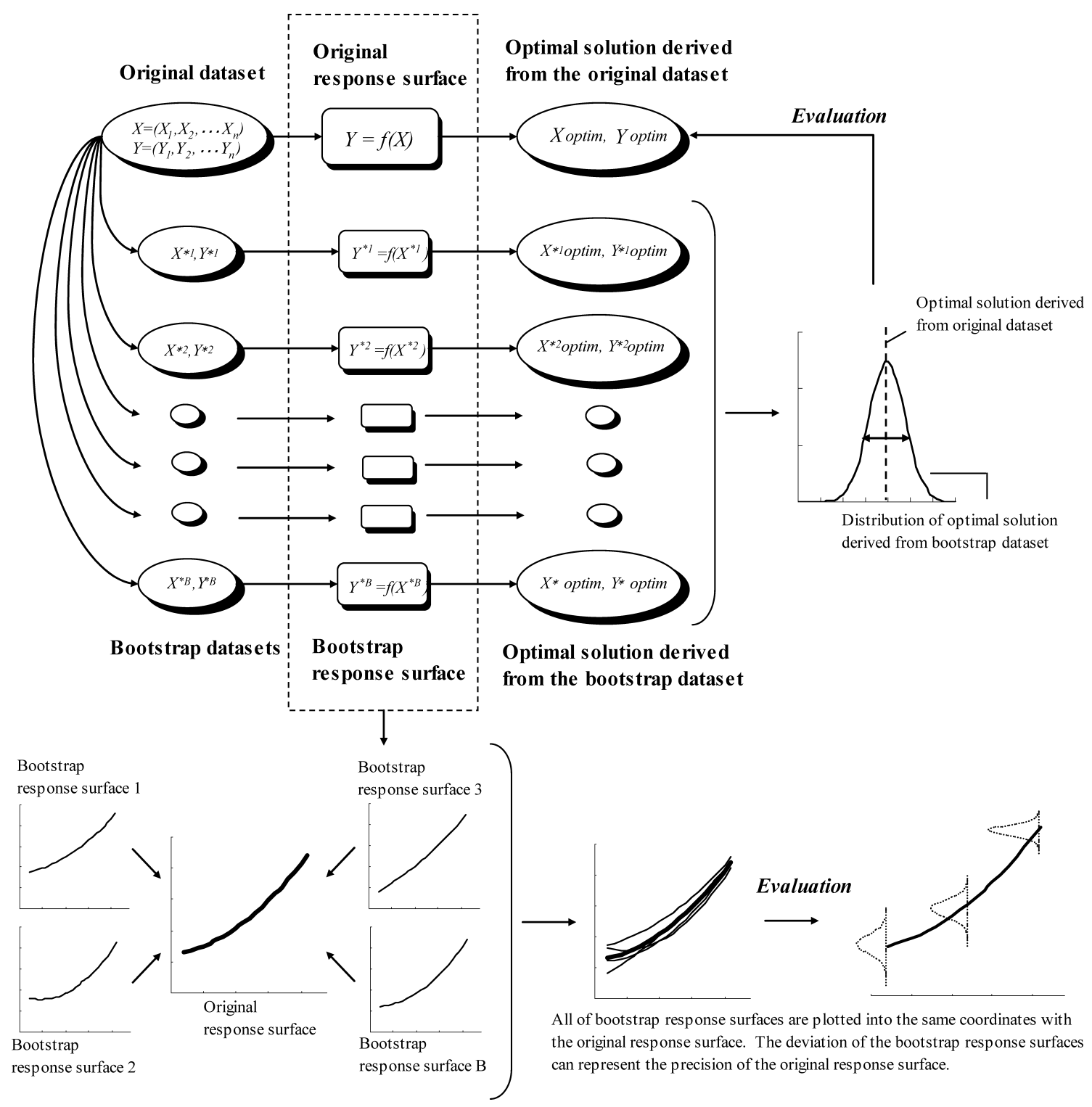

Fig. 1. Evaluation Process for Estimating the Reliability of an Optimal Formulation and Response Surface (Including Design Space) Based on the Bootstrap Resampling Technique

Response Surface Analysis and Simultaneous Optimization of Original Dataset The response surfaces of each response variable were modeled using the $\mathrm{RSM}^{\mathrm{S}}$ method. ${ }^{24)}$ The simultaneous optimum solution was predicted according to the generalized distance function method defined in Eq. $4 .^{25)}$

$$
S(\mathbf{X})=\left\{\sum\left(\frac{F D_{k}(\mathbf{X})-F O_{k}(\mathbf{X})}{S D_{k}}\right)^{2}\right\}^{1 / 2}
$$

where $S(\mathbf{X})$ is the distance function generalized by the standard deviation $S D_{k}$ of the observed values for each response variable, $F D_{k}(\mathbf{X})$ is the optimum value of each response variable optimized individually over the experimental region and $F O_{k}(\mathbf{X})$ is the estimated value of all the responses given in the same set of causal factors, i.e., $\mathbf{X}$. The simultaneous optimum solution can be estimated by minimizing $S(\mathbf{X})$ under the restriction of the experimental region. In this study, we considered the importance of three response variables at the simultaneous optimization were equality. Therefore, the weighting coefficients of these responses were set to the same value.

Evaluation Method for an Optimal Solution and an Acceptance Range Based on BS Technique The reliability of the simultaneous optimal solution and the acceptance range of 3 responses were evaluated by the BS evaluation technique. This process is shown in Fig. 1 and has been described as follows:
Step 1. The BS dataset corresponding to the respective original datasets (comprising $n$ data points) is generated by BS resampling that is repeated $n$ times to form an ensemble comprising $n$ results.

Step 2. Step 1 is repeated $B$ times, and $B$ units of the BS dataset are generated. In this study, the frequency of BS resamplings was set at 100, 200, 300 and 500 .

Step 3. The response surface is modeled for each BS dataset.

Step 4 . The optimal solution is calculated $X^{*}$ optim, $X^{* 1}$ optim, $X^{* 2}$ optim $\cdots X^{* B}$ optim and $Y^{*}$ optim, $Y^{* 1}$ optim, $Y^{* 2}$ optim $\cdots Y^{* B}$ optim from each BS response surface, and the distribution of the optimal solution is generated.

Step 5. The optimal solution and standard deviation of the BS analysis are calculated using Eqs. 5 and 6 as follows:

$$
\begin{aligned}
& F_{B . m}=\frac{1}{B} \sum_{b=1}^{B} F^{* b} \\
& S D_{B}=\left[\frac{1}{B-1} \sum_{b=1}^{B}\left(F^{* b}-F_{B . m}\right)^{2}\right]^{1 / 2}
\end{aligned}
$$

where $F^{* b}$ is the optimal solution of a specified property that is calculated from each BS dataset, $F_{B . m}$ is the BS optimal solution of the same property corresponding to the original solution, and $S D_{B}$ is the standard deviation of the distribution of $F^{* b}$. 
Table 3. Original Experimental Design Dataset of Mefenamic Acid Tablets

\begin{tabular}{|c|c|c|c|c|c|c|}
\hline \multirow{2}{*}{$\begin{array}{c}\text { Experiment } \\
\text { number }\end{array}$} & \multicolumn{3}{|c|}{ Factor level } & \multirow{2}{*}{$\begin{array}{c}\% \text { Dissolved } \\
\text { mefenamic } \\
\text { acid at } 15 \mathrm{~min}, Y_{1}\end{array}$} & \multirow{2}{*}{$\begin{array}{l}\text { Hardness } \\
(\mathrm{N}), Y_{2}\end{array}$} & \multirow{2}{*}{$\begin{array}{c}\text { Particle size }(\mu \mathrm{m}) \\
\text { (median diameter), } \\
Y_{3}\end{array}$} \\
\hline & $X_{1}^{a)}(\%)$ & $X_{2}^{b)}(\mathrm{rpm})$ & $X_{3}^{c)}(\min )$ & & & \\
\hline 1 & 30 & 700 & 10 & $70.4(2.3)$ & $39.2(0.5)$ & $81.1(4.8)$ \\
\hline 2 & 40 & 100 & 10 & $63.2(1.9)$ & $49.2(4.9)$ & $82.6(10.2)$ \\
\hline 3 & 40 & 1300 & 10 & $45.4(1.0)$ & $30.5(0.9)$ & $151.0(8.7)$ \\
\hline 4 & 50 & 700 & 10 & $8.3(1.4)$ & $28.4(5.2)$ & $326.4(14.8)$ \\
\hline 5 & 30 & 100 & 6 & $66.4(0.5)$ & $46.4(1.3)$ & $76.8(7.9)$ \\
\hline 6 & 30 & 1300 & 6 & $67.4(2.3)$ & $40.2(1.6)$ & $90.6(6.8)$ \\
\hline 7 & 50 & 100 & 6 & $63.3(1.5)$ & $30.1(3.5)$ & $114.9(10.3)$ \\
\hline 8 & 50 & 1300 & 6 & $11.6(3.1)$ & $28.8(3.2)$ & $317.6(13.3)$ \\
\hline 9 & 30 & 700 & 2 & $71.5(0.7)$ & $45.4(1.6)$ & $83.3(6.8)$ \\
\hline 10 & 40 & 100 & 2 & $65.2(0.9)$ & $55.4(2.9)$ & $82.3(6.9)$ \\
\hline 11 & 40 & 1300 & 2 & $65.0(0.6)$ & $35.0(3.2)$ & $122.1(15.0)$ \\
\hline 12 & 50 & 700 & 2 & $22.8(2.8)$ & $45.4(8.1)$ & $245.8(12.9)$ \\
\hline 13 & 40 & 700 & 6 & $51.4(5.7)$ & $43.7(2.7)$ & $136.3(11.7)$ \\
\hline 14 & 40 & 700 & 6 & $42.9(2.8)$ & $49.9(3.7)$ & $138.7(15.9)$ \\
\hline
\end{tabular}

( ): Standard deviation for individual values of 3 determinations. a) Amount of water added (\%). b) Impeller rotation speed (rpm). c) Kneading time (min).
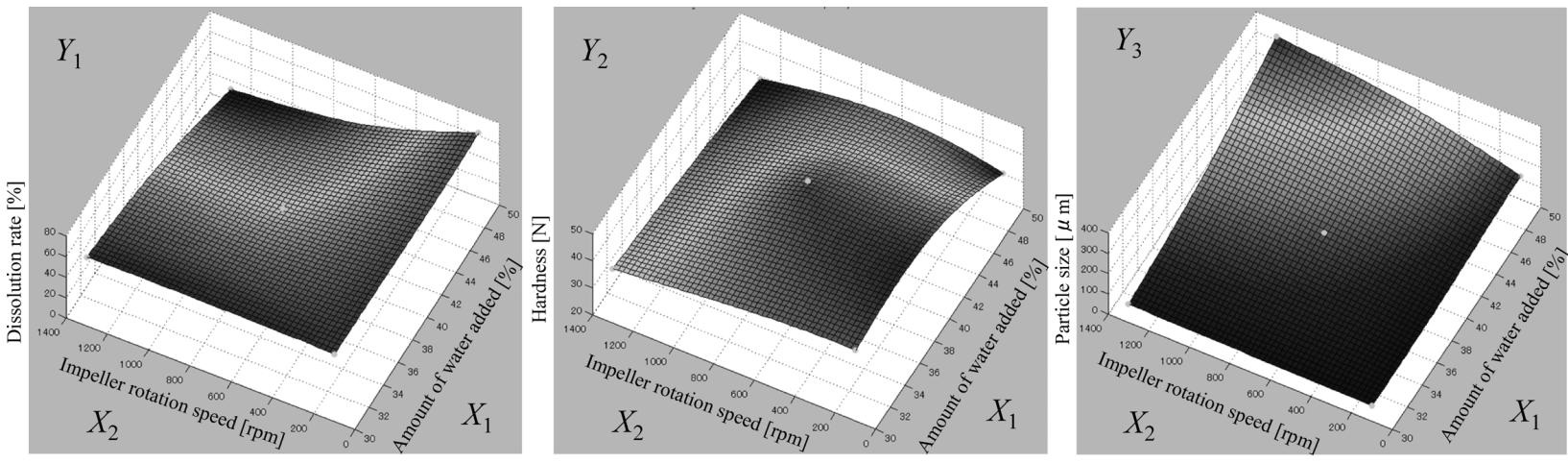

Fig. 2. The Response Surfaces of Three Target Variables ( $Y_{1}: \%$ Dissolved Mefenamic Acid at 15 min, $Y_{2}$ : Hardness of Core Tablets, and $Y_{3}$ : Particle Size (Median Diameter) of Screened Granules as a Function of the Amount of Water Added $\left(X_{1}\right)$ and the Impeller Rotation Speed $\left(X_{2}\right)$ at a Constant $\operatorname{Kneading}$ Time $\left(\mathrm{X}_{3}: 6\right.$ min $\left.)\right)$

Step 6. The response surfaces, modeled from $B$ units of the BS dataset, are plotted in a multidimensional space, and the standard deviation of the predicted solution is calculated for all grids of the space.

The accuracy of the original solution, which was calculated from the original dataset, can be evaluated by comparing it with the BS solutions. If the accuracy of a BS solution deviates from that of the original solution, the original solution is considered to have low reliability with regard to accuracy. The precision of the original solution can be evaluated using the standard deviation of BS optimal solutions. Moreover, the reliability of the response surface can also be evaluated from the standard deviation of the predicted solution in each grid of the space. A large standard deviation indicates poor precision of the response surface model and the optimal solution.

Evaluation Indices of Accuracy for Optimal Solutions The value of $d$ used as the evaluation index of accuracy of the optimal solution was calculated using Eq. 7. This value represents the similarity between the original solution and the BS optimal solution.

$$
d=\frac{\left|F-F_{B . m}\right|}{F} \times 100
$$

where $F$ is the original solution of a specified property, and $F_{B . m}$ is the BS optimal solution of the same property corresponding to the original solution.

Software JMP ${ }^{\mathrm{TM}} 8$ (SAS institute Inc., U.S.A.) was used for preparing the DOE and the statistical analysis. dataNESIA ${ }^{\mathrm{TM}}$ (Yamatake Corp., Japan) was used for generating the response surface and estimating the optimal solution. This software consists of a multidimensional spline interpolation program and a nonlinear optimization program. ${ }^{24}$ ) Viscovery ${ }^{\circledR}$ (Eudaptics Software Gmbh, Austria) was used for SOM clustering. This software can order complex data based on similarity. The ordered data are separated into clusters on the basis of similarity, and these clusters are shown on a multi-colored map. The resulting map can be used to extract the features hidden in the data. Voxler ${ }^{\circledR}$ (Golden software Inc., U.S.A.) was used for the multi-dimensional visualization of the response surface models.

\section{Results and Discussion}

Response Surface Modeling by $\mathbf{R S M}^{\mathbf{S}}$ The dissolution ratio of mefenamic acid for the first $15 \mathrm{~min}\left(Y_{1}\right)$, hardness of the core tablets $\left(Y_{2}\right)$, and particle size (median diameter) of the screened granules $\left(Y_{3}\right)$ of each formulation are listed in Table 3. These parameters varied across the manufacturing conditions. The reproducibility for individual values of $3 \mathrm{de}-$ terminations was fairly good. The response surfaces of $Y_{1}$, $Y_{2}$, and $Y_{3}$ were generated by $\mathrm{RSM}^{\mathrm{S}}$ as functions of 3 causal factors: the amount of water added $\left(X_{1}\right)$, impeller rotation speed $\left(X_{2}\right)$, and kneading time $\left(X_{3}\right)$ in the granulation process. Representative examples of the response surfaces are shown in Fig. 2. These response surfaces showed $X_{1}$ and $X_{2}$ that were interactively affected for three target variables. From this examples, we considered that the increment of the water amount $\left(X_{1}\right)$ and impeller rotation speed $\left(X_{2}\right)$ made the granules size $\left(Y_{3}\right)$ large and stiff, and the dissolution property $\left(Y_{1}\right)$ and hardness $\left(Y_{2}\right)$ decreased. The reliability of each response surface was estimated by using a conventional leaveone-out cross-validation (LOOCV) method. These results are shown in Fig. 3. The values of the correlation coefficient $(r)$ were approximately $0.5-0.7$, which suggested that the reliability of all the response surfaces was poor. The shapes of these response surfaces (for example, as shown in Fig. 2) were rather complicated, indicating that the effect of individual data points in the response surface model was sufficiently 


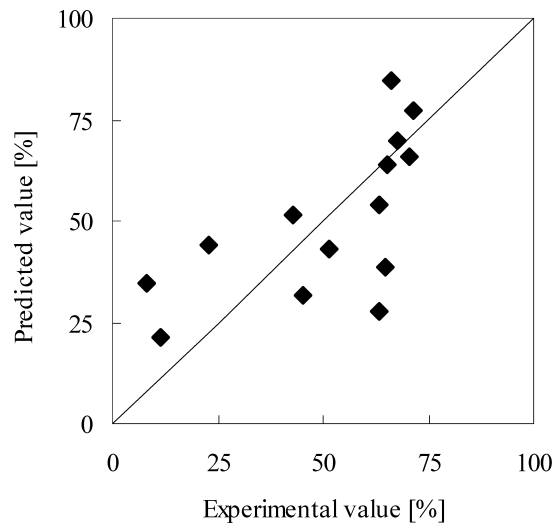

[Y $Y_{1}$ : \%Dissolved mefenamic acid at $15 \mathrm{~min}$ $(r: 0.65)$

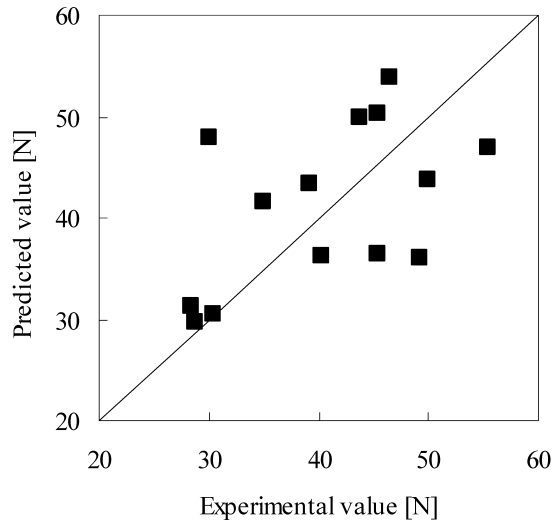

$\left[Y_{2}\right]$ Hardness $(r: 0.52)$

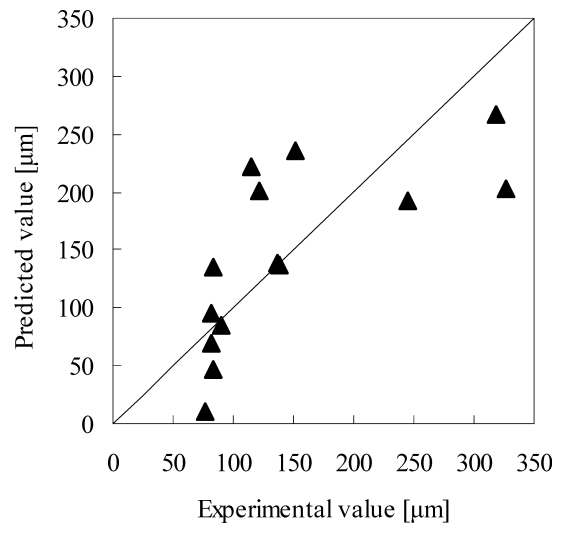

$\left[Y_{3}\right]$ Particle size (median diameter) $(r: 0.70)$

$*_{r}$ : Correlation coefficient

Fig. 3. Relationships between Experimental and Predicted Values of Three Target Variables $\left(Y_{1}\right.$ : \% Dissolved Mefenamic Acid at 15 min, $Y_{2}$ : Hardness of Core Tablets, and $Y_{3}$ : Particle Size (Median Diameter) of Screened Granules)

Table 4. Optimum Formulations of Three Response Variables and Simultaneous Optimum Formulation Predicted by RSM ${ }^{\mathrm{S}}$

\begin{tabular}{|c|c|c|c|c|c|c|}
\hline & \multicolumn{3}{|c|}{ Optimized formulations } & \multicolumn{3}{|c|}{ Predicted responses } \\
\hline & $X_{1}^{a)}(\%)$ & $X_{2}^{b)}(\mathrm{rpm})$ & $X_{3}^{c)}(\min )$ & $Y_{1}^{d)}(\%)$ & $Y_{2}^{e)}(\mathrm{N})$ & $Y_{3}^{f)}(\mu \mathrm{m})$ \\
\hline Optimum formulation of $Y_{1}$ & 30.1 & 732 & 5.6 & 68.0 & 44.5 & 82 \\
\hline Optimum formulation of $Y_{2}$ & 38.6 & 298 & 3.1 & 61.4 & 51.1 & 97 \\
\hline Optimum formulation of $Y_{3}$ & 48.9 & 922 & 7.1 & 19.0 & 32.5 & 276 \\
\hline Simultaneous optimum formulation & 43.7 & 700 & 2.3 & 45.0 & 46.2 & 166 \\
\hline
\end{tabular}

a) Amount of water added. b) Impeller rotation speed. c) Kneading time. d) \% Dissolved mefenamic acid at 15 min. e) Hardness. f) Particle size (median diameter).
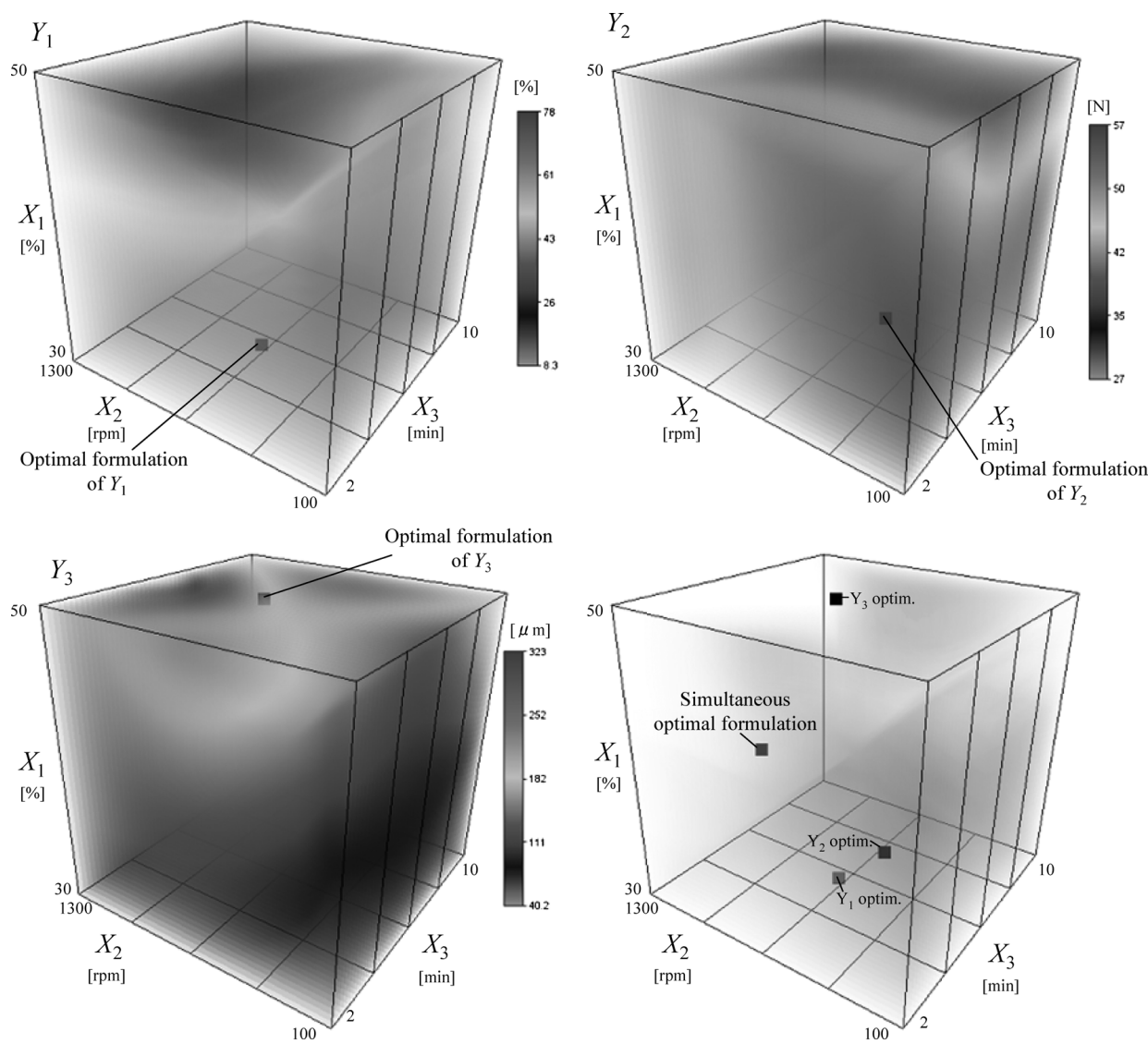

Fig. 4. Response Spaces and Optimum Formulations of Three Target Variables $\left(Y_{1}: \%\right.$ Dissolved Mefenamic Acid at 15 min, $Y_{2}$ : Hardness of Core Tablets, and $Y_{3}$ : Particle Size (Median Diameter) of Screened Granules), and Simultaneous Optimum Formulation Predicted by RSM ${ }^{\mathrm{S}}$ 
Table 5. Original Solution, Bootstrap Optimal Solution, Bootstrap Standard Deviation, and 95\% Confidence Interval of Optimal Solutions Generated by Different Frequencies of Bootstrap Resampling

\begin{tabular}{|c|c|c|c|c|c|c|}
\hline \multirow{2}{*}{$\begin{array}{l}\text { Resampling } \\
\text { frequency }\end{array}$} & \multicolumn{3}{|c|}{ Optimized formulations } & \multicolumn{3}{|c|}{ Predicted responses } \\
\hline & $X_{1}^{b)}(\%)$ & $X_{2}^{c)}(\mathrm{rpm})$ & $X_{3}^{d)}(\min )$ & $Y_{1}^{e)}(\%)$ & $Y_{2}^{f)}(\mathrm{N})$ & $Y_{3}^{g)}(\mu \mathrm{m})$ \\
\hline$N=0^{a)}$ & 43.7 & 700 & 2.3 & 45.0 & 46.2 & 166 \\
\hline \multirow[t]{2}{*}{$N=100$} & $41.2(2.8)$ & $671(172)$ & $2.4(1.3)$ & $50.9(6.3)$ & $46.8(3.0)$ & $145(22)$ \\
\hline & {$\left[\begin{array}{lll}33.9 & 45.4\end{array}\right]$} & {$[111-957]$} & {$[2.0-6.1]$} & {$[41.8-62.8]$} & {$[41.4-53.5]$} & {$[103-181]$} \\
\hline \multirow[t]{2}{*}{$N=200$} & $41.5(2.6)$ & $677(155)$ & $2.5(1.3)$ & $49.8(6.3)$ & $46.5(2.8)$ & $148(23)$ \\
\hline & {$\left[\begin{array}{ll}36.0 & 46.2\end{array}\right]$} & {$[208-914]$} & {$[2.0-6.0]$} & {$[40.5-64.1]$} & {$[41.8-53.1]$} & [99-184] \\
\hline \multirow[t]{2}{*}{$N=300$} & $41.4(2.5)$ & $673(167)$ & $2.4(1.1)$ & $50.1(6.1)$ & $46.6(3.2)$ & $148(20)$ \\
\hline & {$[35.5-45.6]$} & {$[130-914]$} & {$[2.0-6.0]$} & {$[42.6-65.2]$} & {$[41.9-53.2]$} & {$[101-181]$} \\
\hline \multirow[t]{2}{*}{$N=500$} & $41.3(2.7)$ & $677(165)$ & $2.4(1.2)$ & $50.6(6.3)$ & $46.6(2.9)$ & $146(22)$ \\
\hline & {$[34.9-45.4]$} & {$[111-957]$} & {$[2.0-6.0]$} & {$[41.3-64.6]$} & {$[41.9-53.4]$} & {$[100-182]$} \\
\hline
\end{tabular}

( ): Bootstrap standard deviation. [ ]: 95\% confidence interval calculated by percentile method. a) Obtained from the original dataset. $b$ ) Amount of water added. c) Impeller rotation speed. d) Kneading time. e) \% Dissolved mefenamic acid at 15 min. $f$ ) Hardness. $g$ ) Particle size (median diameter).

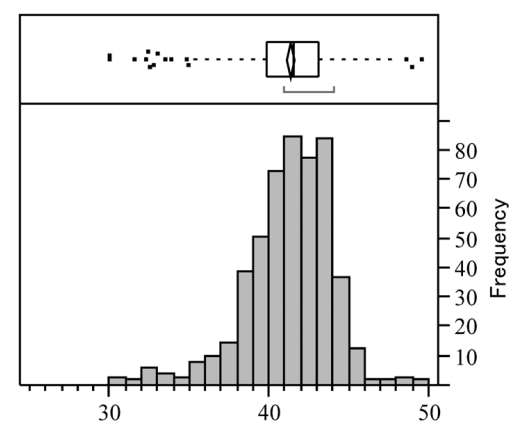

Amount of water added [\%]

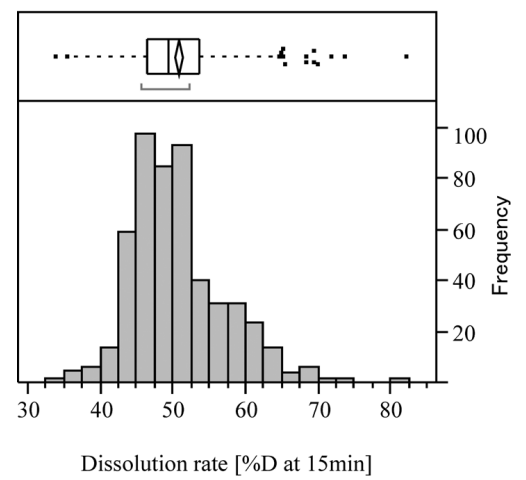

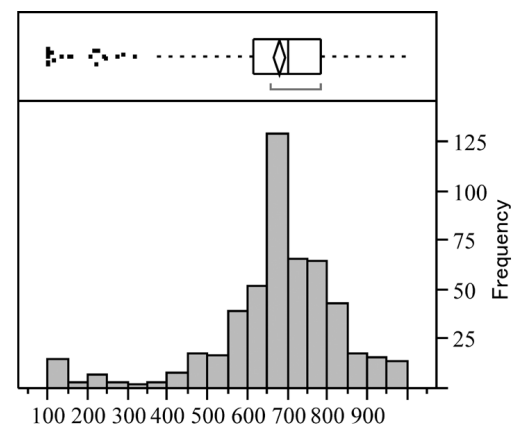

Impeller rotation speed [rpm]

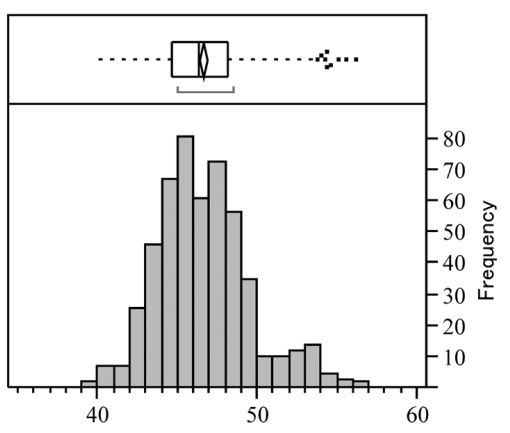

Hardness $[\mathrm{N}]$

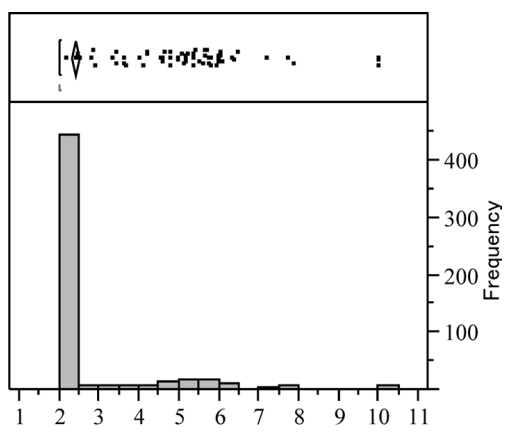

Kneading time [min]

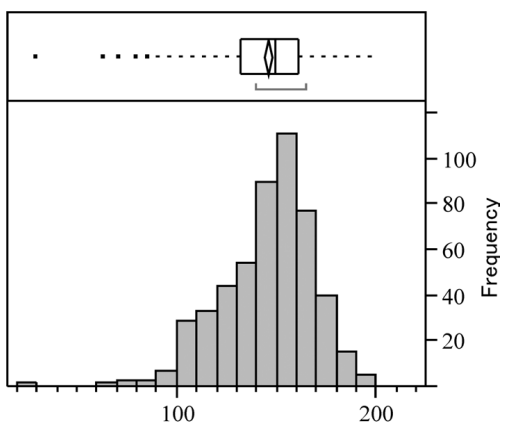

Particle size (median diameter) $[\mu \mathrm{m}]$

Fig. 5. Histograms of Optimal Solutions Generated by Bootstrap Resampling $(N=500)$

high. Therefore, the response surface was considered to be widely changed by leaving out individual experimental data in the LOOCV process.

Prediction of the Optimal Formulation by $\operatorname{RSM}^{\mathrm{S}}$ and Generalized Distance Function Method The optimal formulation of three responses for $X_{1}, X_{2}$, and $X_{3}$, and the predicted values for $Y_{1}, Y_{2}$, and $Y_{3}$ calculated by $\mathrm{RSM}^{\mathrm{S}}$ and generalized distance function method are shown in Table 4. The simultaneous optimal solutions for $X_{1}, X_{2}$, and $X_{3}$, and the predicted values for $Y_{1}, Y_{2}$, and $Y_{3}$ are additionally shown in Table 4. Moreover, the correlation of these results are visually described in Fig. 4. The optimal formulation of each response sufficiently maximized the target variables. However, these optima were not appropriate conditions for the other targets. On the other hand, the simultaneous optimal formulation predicted by the generalized distance function method adequately maximized the three targets.

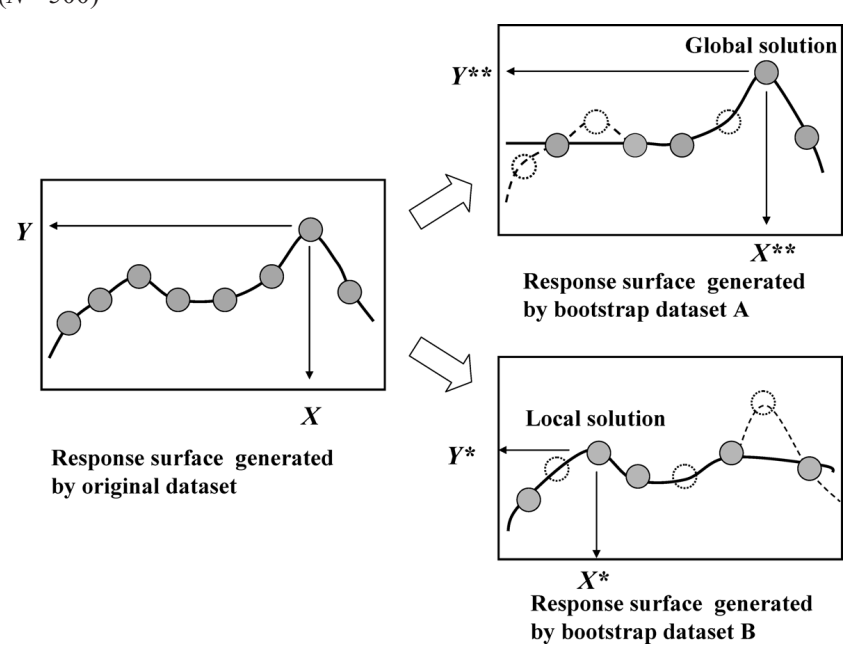

Fig. 6. The Reason for Mingling the Global Solution and Local Solutions in the Bootstrap Resampling Process 
Table 6. Bootstrap Optimal Solutions and Bootstrap Standard Deviations in the Self-Organizing Map Clusters for Optimal Solution Estimated from Bootstrap Samples

\begin{tabular}{|c|c|c|c|c|c|c|}
\hline & \multicolumn{3}{|c|}{ Optimized formulations } & \multicolumn{3}{|c|}{ Predicted responses } \\
\hline & $X_{1}^{c)}(\%)$ & $X_{2}^{d)}(\mathrm{rpm})$ & $X_{3}^{e)}(\min )$ & $Y_{1}^{f)}(\%)$ & $Y_{2}^{g)}(\mathrm{N})$ & $Y_{3}^{h)}(\mu \mathrm{m})$ \\
\hline Original solution $^{a)}$ & 43.7 & 700 & 2.3 & 45.0 & 46.2 & 166 \\
\hline \multicolumn{7}{|l|}{ Bootstrap solution $^{b)}$} \\
\hline Cluster 1 & 42.4 & 678 & 2.0 & 48.9 & 46.5 & 153 \\
\hline (324 data) & $(1.5)$ & $(106)$ & $(0.1)$ & $(5.0)$ & $(2.5)$ & $(18)$ \\
\hline Cluster 2 & 38.3 & 783 & 2.1 & 56.0 & 46.5 & 130 \\
\hline (96 data) & $(2.2)$ & $(110)$ & $(0.3)$ & $(6.9)$ & $(3.1)$ & $(25)$ \\
\hline Cluster 3 & 38.8 & 724 & 5.6 & 51.7 & 45.3 & 130 \\
\hline (54 data) & $(2.6)$ & $(114)$ & $(1.4)$ & $(7.0)$ & $(1.8)$ & $(22)$ \\
\hline Cluster 4 & 44.1 & 166 & 2.0 & 49.7 & 51.3 & 148 \\
\hline (26 data) & $(2.7)$ & (79) & $(0.0)$ & $(5.8)$ & $(3.3)$ & $(22)$ \\
\hline
\end{tabular}

( ): Bootstrap standard deviation. a) Obtained from the original dataset. b) Bootstrap resampling frequency, 500 times. c) Amount of water added. d) Impeller rotation speed. e) Kneading time. f) \% Dissolved mefenamic acid at 15 min. g) Hardness. $h$ ) Particle size (median diameter).

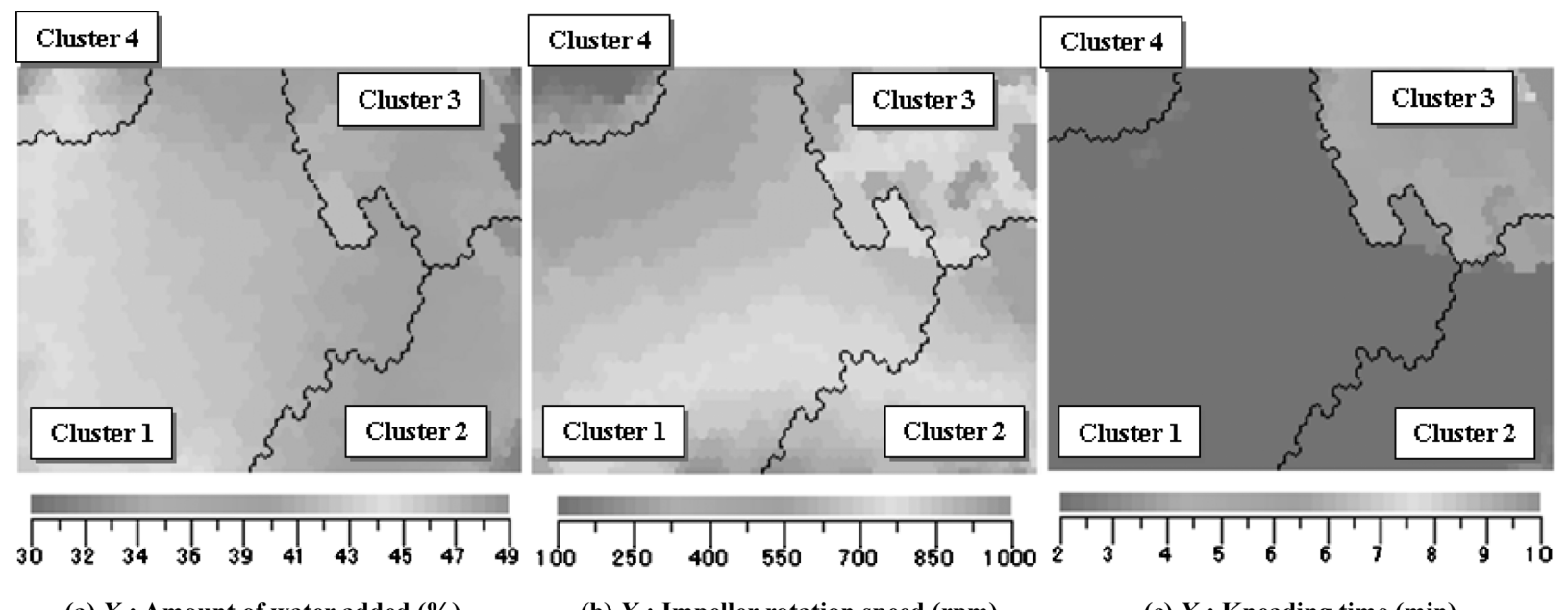

(a) $X_{1}$ : Amount of water added (\%)

(b) $X_{2}$ : Impeller rotation speed $(\mathbf{r p m})$

(c) $X_{3}$ : Kneading time (min)

Fig. 7. Self-Organizing Maps of Optimal Solutions for Input Factors Estimated from Bootstrap Samples $(N=500)$

\section{Evaluation of the Optimal Formulation by the BS}

Method BS datasets were generated by BS resampling that was set at a frequency of 100, 200, 300, and 500. The results of the optimal formulations and the predicted responses with a 95\% confidence interval are shown in Table 5. The BS optimal solutions as well as the standard deviations were almost constant despite altering the resampling frequency. Therefore, we considered that a resampling frequency of more than 100 was sufficient to estimate the stability of the optimal formulations. The distributions of the BS optimal solutions when bootstrapping was repeated 500 times are shown in Fig. 5. The BS optimal solutions were moderately the same as the original solution. The distributions of the BS optimal solutions were almost symmetrical. However, several solutions that had been quite different from the original solution were contained. We previously reported that this problem was the risk involved in the BS resampling process, as shown in Fig. 6. ${ }^{26)}$ As mentioned above, BS datasets are generated from the original data. If the corrected data points are located near the original solution, the BS optimal solution is consistent with the global optima. On the other hand, if the corrected data points are far from the original solution, the BS optimal solution may lead to a local optima. Therefore, we interpret the problem as easily occurring when the relation-

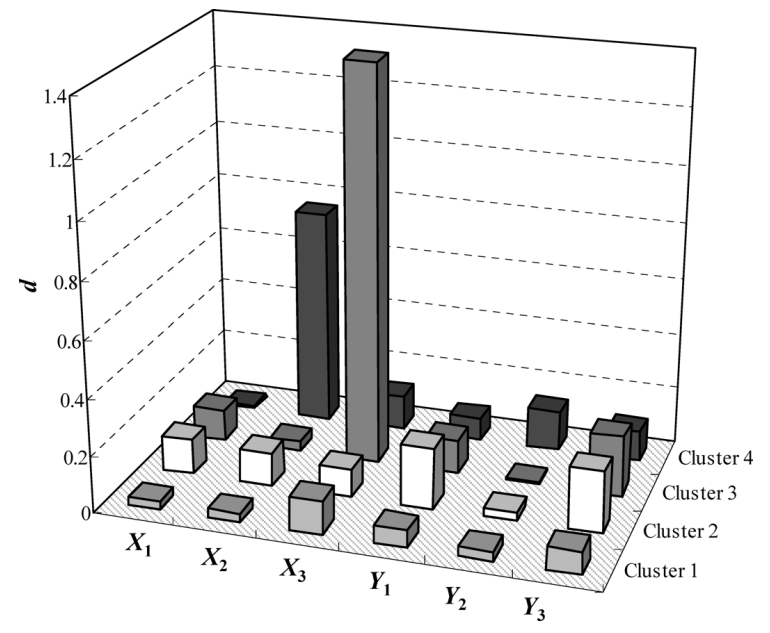

Fig. 8. Comparison of the $d$ Index to Determine the Accuracy of the Optimal Solution in the Self-Organizing Map Clusters

ships between causal factors and responses are markedly nonlinear. Consequently, it was considered that global and local solutions were mixed in the distributions of BS solutions as shown in Fig. 5. 
To resolve this problem, SOM clustering was applied for dividing the global and the local solutions. The distributions of the optimal solutions were classified by SOM clustering. The SOMs of the BS optimal formulations of each causal factor are shown in Fig. 7. In the SOM analysis, input variables of three causal factors were standardized by the standard deviation of each factor. The BS optimal formulations were classified into 4 clusters, as shown in Table 6 . Then, the $d$ index corresponding to the accuracy of each optimal solution was calculated using Eq. 7 in each cluster (Fig. 8). The BS optimal solution in the case of cluster 1 was similar to the original solution. Moreover, the $d$ values of cluster 1 were apparently smaller than those of the other clusters. A small $d$ value indicates high accuracy. Therefore, the global optimal solution was considered to be contained in the BS optimal solutions of cluster 1 . The histograms of the optimal solutions in cluster 1 are shown in Fig. 9. These distribution ranges were almost narrower than those of the original distributions (shown in Fig. 5). This indicated that the SOM could satisfactorily separate the mixture of the BS optimal solutions into several groups.

In the previous study, the number of BS resamplings re-

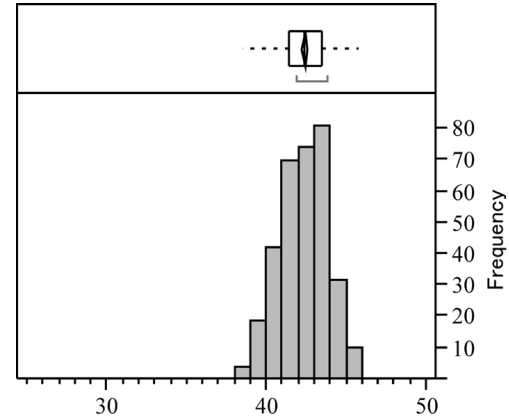

Amount of water added [\%]

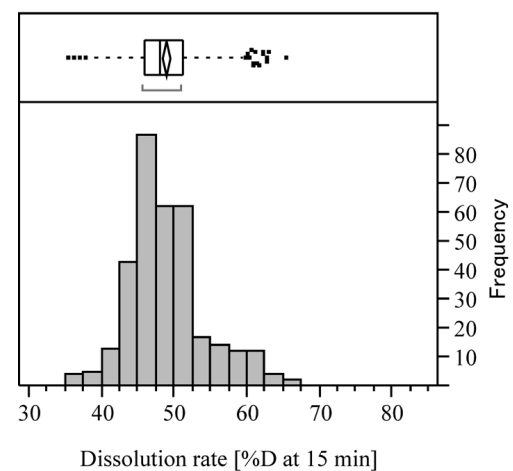

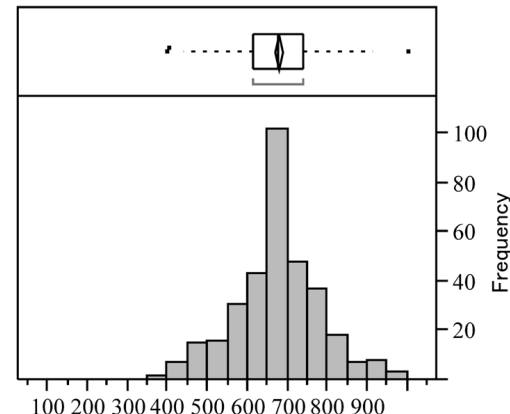

Impeller rotation speed [rpm]

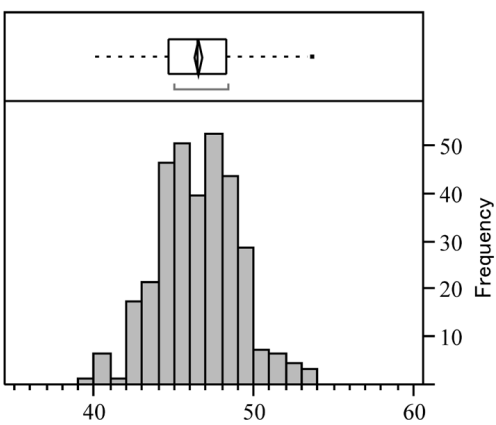

Hardness [N]

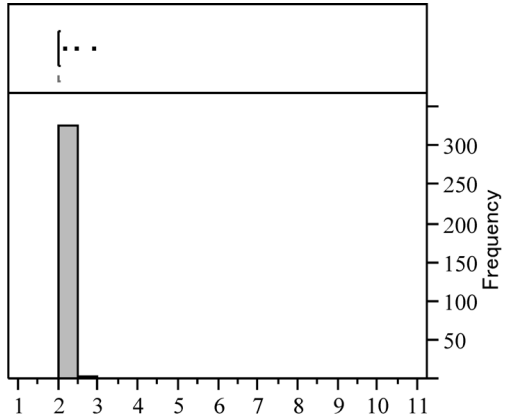

Kneading time [min]

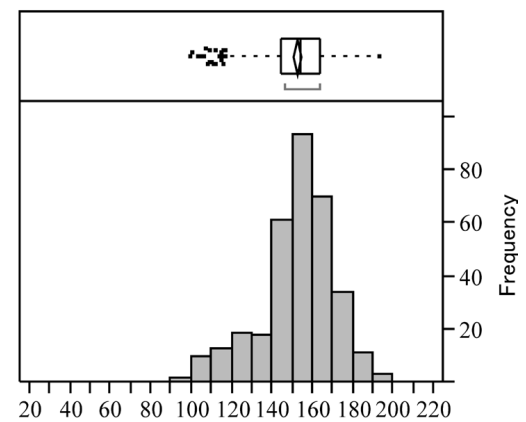

Particle size (median diameter) $[\mu \mathrm{m}]$

Fig. 9. Histograms of Optimal Solutions in Cluster 1 Separated by Self-Organizing Map Clustering of the 500 Bootstrappings of the Original Dataset

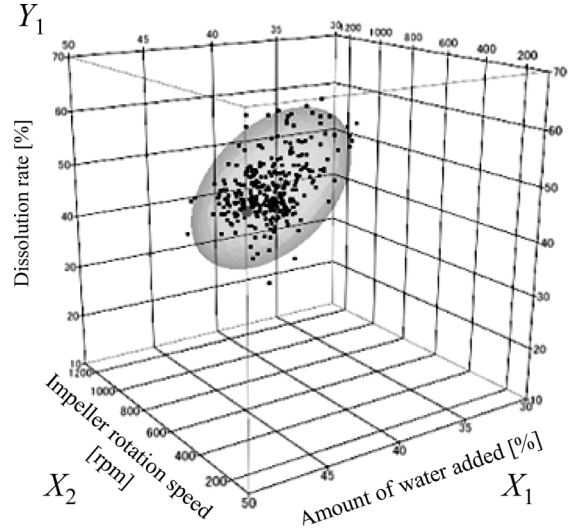

[Y $Y_{1}$ : \%Dissolved mefenamic acid at $15 \mathrm{~min}$

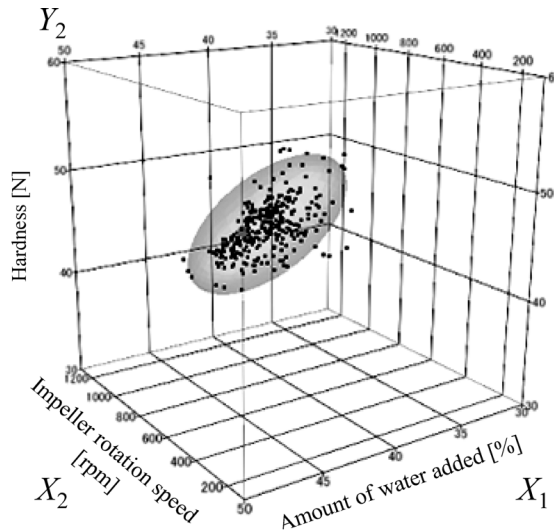

$\left[Y_{2}\right]$ Hardness

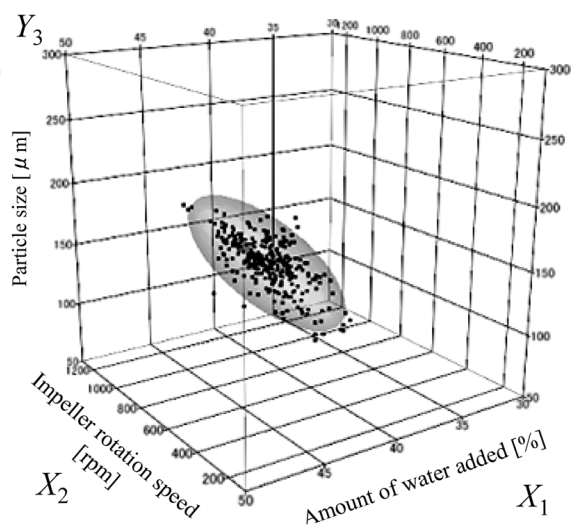

$\left[Y_{3}\right]$ Particle size (median diameter)

:Original optimal formulation

Bootstrap optimal formulations

:Normal contour ellipsoid of bootstrap solution (coverage:95\%)

Fig. 10. Statistical Graphics of the Bootstrap Optimal Solutions in Cluster 1 Separated by Self-Organizing Map Clustering of the 500 Bootstrappings of the Original Dataset ( $Y_{1}: \%$ Dissolved Mefenamic Acid at $15 \mathrm{~min}, Y_{2}$ : Hardness of Core Tablets, and $Y_{3}$ : Particle Size (Median Diameter) of Screened Granules) as a Function of the Amount of Water Added $\left(X_{1}\right)$ and the Impeller Rotation Speed $\left(X_{2}\right)$ ) 

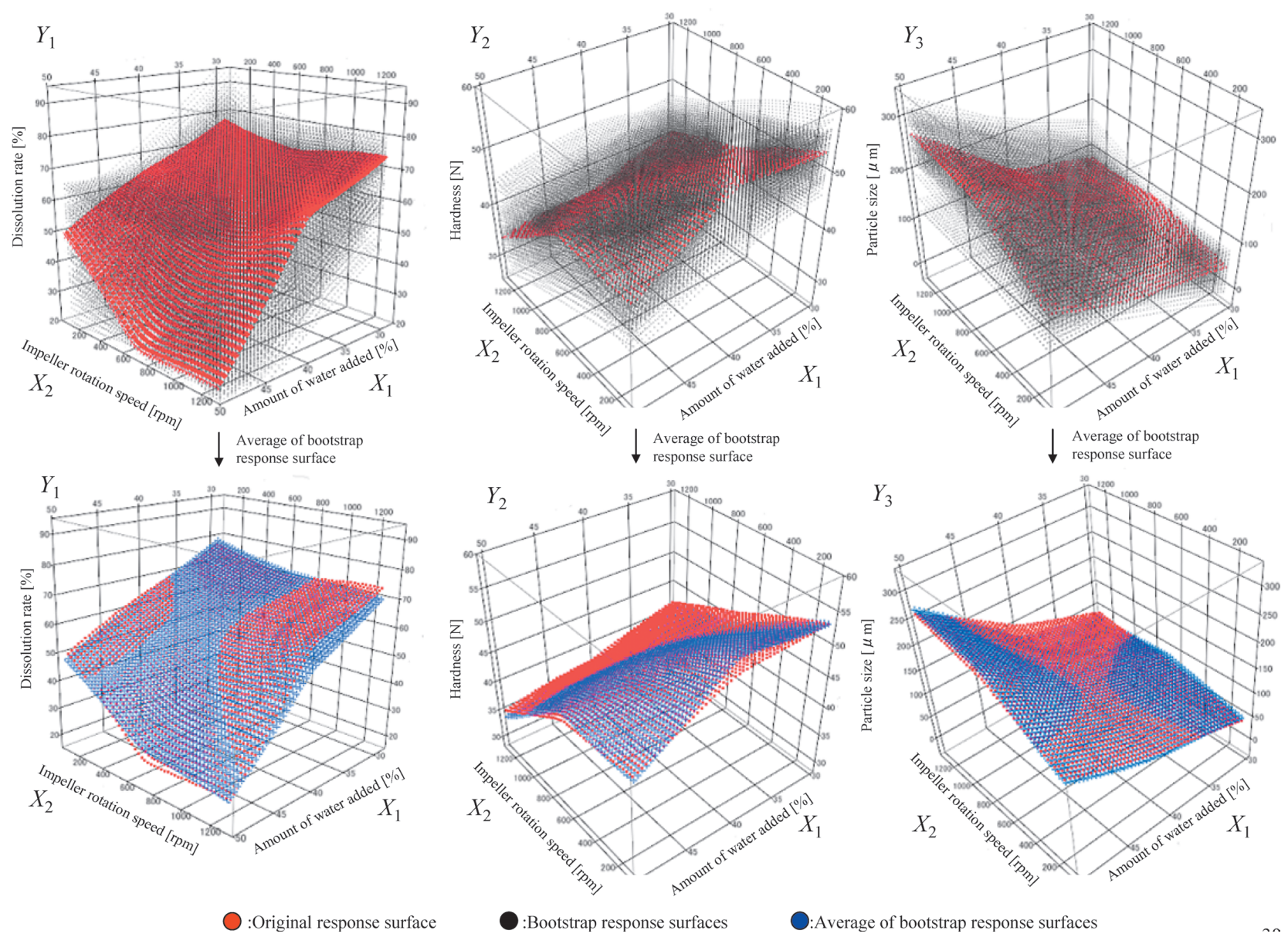

Fig. 11. The Original Response Surfaces, Bootstrap Response Surfaces, and Averages of Bootstrap Response Surfaces of Three Target Variables ( $Y_{1}$ : \% Dissolved Mefenamic Acid at $15 \mathrm{~min}, Y_{2}$ : Hardness of Core Tablets, and $Y_{3}$ : Particle Size (Median Diameter) of Screened Granules) as a Function of the Amount of Water Added $\left(X_{1}\right)$ and the Impeller Rotation Speed $\left(X_{2}\right)$ at a Constant Kneading Time $\left(X_{3}: 2.3 \mathrm{~min}\right)$ )

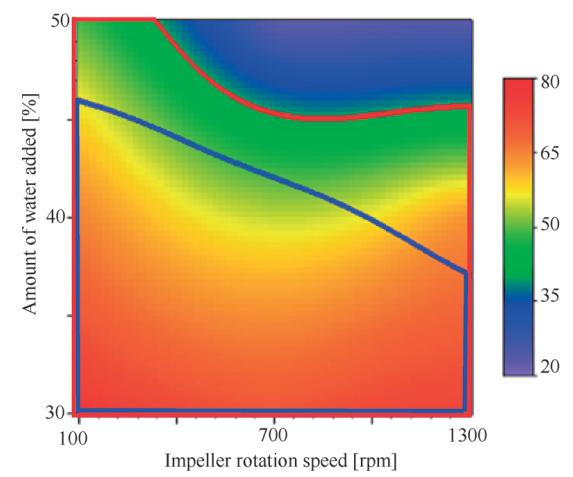

$Y_{1}: \%$ Dissolved mefenamic acid at $15 \mathrm{~min}$ (Acceptance criteria: NLT 40\%)

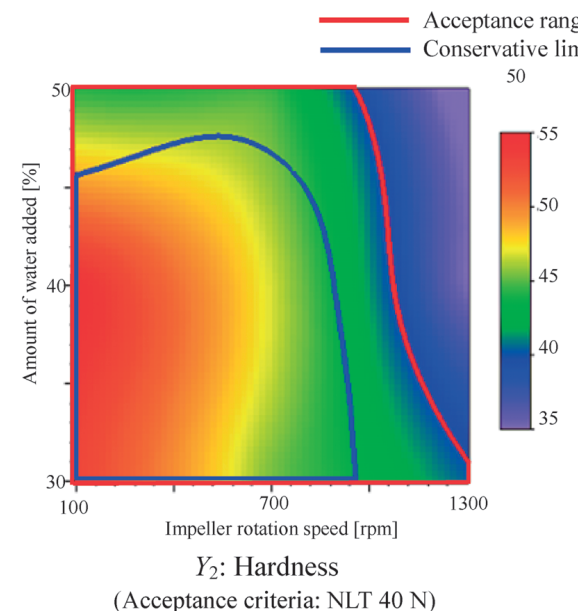

(Acceptance criteria: NLT $40 \mathrm{~N}$ ) tance range derived from original response surface
ervative limit of the $95 \%$ confidence interval of acceptance range

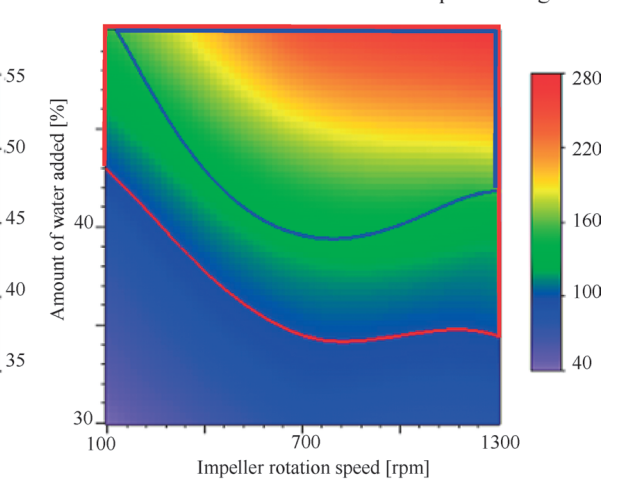

$Y_{3}$ : Particle size (median diameter) (Acceptance criteria: NLT $100 \mu \mathrm{m}$ )

Fig. 12. The Design Space of Three Target Variables Derived from the Original Response Surfaces and Those of the Conservative Limit of the 95\% Confidence Interval ( $Y_{1}: \%$ Dissolved Mefenamic Acid at $15 \mathrm{~min}, Y_{2}$ : Hardness of Core Tablets, and $Y_{3}$ : Particle Size (Median Diameter) of Screened Granules) as a Function of the Amount of Water Added $\left(X_{1}\right)$ and the Impeller Rotation Speed $\left(X_{2}\right)$ at a Constant Kneading Time $\left(X_{3}: 2.3\right.$ min $)$

quired for the evaluation of the optimal solution was at least around $300 .{ }^{16)}$ Therefore, we considered cluster 1 to be appropriate for determining the reliability of the response surface and optimal solution calculated from the original dataset. For the representative examples of BS analysis, the normal contour ellipsoids of three responses are shown in
Fig. 10. The original optimal formulation are almost plotted in the middle of the normal counter ellipsoids, and we could visually evaluate the fluctuation of the predicted solutions from the dimensions of the ellipsoids.

Evaluation of the Design Space by the BS Technique in Combination with SOM Clustering The response sur- 
faces of the 3 response variables $Y_{1}, Y_{2}$, and $Y_{3}$ modeled from all the BS datasets contained in cluster 1 are plotted in the same coordinates with the original response surface. Representative examples of these results are shown in Fig. 11. The plots of BS response surfaces are distributed around the original response surfaces. Moreover, the average of all BS response surfaces sufficiently corresponded with the original response surface (shown in Fig. 11). These results support the hypothesis that the novel method based on a BS resampling technique can be applied for evaluating the reliability of the original response surface and optimal solution.

Conservative limits of the $95 \%$ confidence intervals of the acceptance ranges in 3 response variables were calculated using the standard deviations of the BS response surfaces. Examples of the results are shown in Fig. 12. These figures clearly show the difference between the acceptance range derived from the original response surface and the robust design space estimated by the conservative limits of the $95 \%$ confidence intervals of the acceptance ranges, statistically.

\section{Conclusion}

It was confirmed that a novel method based on BS resampling and SOM was applicable for evaluating the precision of a nonlinear response surface. Thus, the novel method described in this study was considered to be useful as a science-based rationale of the design space.

Acknowledgements The authors are very grateful to Mr. Takayuki Otsuki, Mr. Kazuhisa Sekine, and Ms. Yasuko Abe, Daiichi Sankyo Co., for their assistance in the experimental work.

\section{References}

1) ICH guideline, "Pharmaceutical Development Q8 (R2)," August 2009.

2) Huang J., Kaul G., Cai C. S., Chatlapalli R., Hernendez-Abad P., Ghosh K., Nagi A., Int. J. Pharm., 382, 23-32 (2009).
3) Montgomery D. C., "Design and Analysis of Experiments," John Wiley \& Sons, U.S.A., 1997.

4) Lewis G. A., Mathieu D., Phan-Tan-Luu R., "Pharmaceutical Experimental Design,” Marcel Dekker, New York, 1999.

5) Rekhi G. S., Nellore R. V., Hussain A. S., Tillman L. G., Malinowski H. J., Augsburger L. L., J. Controlled Release, 59, 327-342 (1999).

6) Paterakis P. G., Korakianiti E. S., Dallas P. P., Rekkas D. M., Int. J. Pharm., 248, 51-60 (2002).

7) Huang Y. B., Tasai Y. H., Yang W. C., Chang J. S., Wu P. C., Biol. Pharm. Bull., 27, 1626-1629 (2004).

8) Aikhatib H. S., Sakr A., Pharm. Dev. Technol., 8, 87-96 (2003).

9) Marengo E., Cavalli R., Rovero G., Gasco M. R., Pharm. Dev. Technol., 8, 299-309 (2003).

10) Matsumura M., Nakagami H., Yamao T., Takayama K., Nagai T., Chem. Pharm. Bull., 42, 1902-1908 (1994).

11) Vojnovic D., Chicco D., Zenary H. El., Int. J. Pharm., 145, 203-213 (1996).

12) Takayama K., Morva A., Fujikawa M., Hattori Y., Obata Y., Nagai T., J. Controlled Release, 68, 175-186 (2000).

13) Wu P. C., Obata Y., Fujikawa M., Li C. J., Higashiyama K., Takayama K., J. Pharm. Sci., 90, $1004-1014$ (2001).

14) Takayama K., Obata Y., Morishita M., Nagai T., Pharmazie, 59, 392 395 (2004).

15) Efron B., Tibshirani R. J., "An Introduction to the Bootstrap," Chapman and Hall, New York, 1993.

16) Arai H., Suzuki T., Kaseda C., Ohyama K., Takayama K., Chem. Pharm. Bull., 55, 586-593 (2007).

17) Kohonen T., Proc. IEEE, 78, 1464-1480 (1990).

18) Arai H., Suzuki T., Kaseda C., Takayama K., Chem. Pharm. Bull., 57, $572-579$ (2009).

19) Kallel R., Cottrell M., Vigneron V., Neurocomputing, 48, 175-183 (2002).

20) Ueda N., Nakano R., IEEE Int. Conf. Neural Netw. Proc., 1, 101-104 (1995).

21) Dupret G., Koda M., Eur. J. Oper. Res., 134, 141-156 (2001).

22) Zhang J., Neural Networks, 12, 927 -938 (1999).

23) Noriega G., Neural Networks, 21, 130-139 (2008).

24) Kaseda C., Kagaku Kogaku, 68, 315-317 (2004).

25) Takayama K., Nagai T., Int. J. Pharm., 74, 115-126 (1991).

26) Onuki Y., Ohyama K., Kaseda C., Arai H., Suzuki T., Takayama K., J. Pharm. Sci., 97, 331-339 (2008). 